A $\mathrm{N}$ article by C. L. Crawford (Nature, March 20), stressed in vigorous terms the well recognised concept that the best available means of secondary prevention of leprosy lies in the regular and sustained treatment of leprosy sufferers with the cheap and effective mycobacteriostatic drug, dapsone. We agree, and have said the same, many times.

The well-night insuperable difficulties facing the World Health Organisation and voluntary agencies, in trying to implement a strategy on which they are now generally agreed, are concerned with factors to which Crawford seems to pay little attention in his article. These factors are, on the one hand, the social aspects of leprosy, their effect on government policy and priorities, and patient attitudes to leprosy, and, on the other, the inadequacy of diagnostic and curative facilities in precisely those countries where leprosy constitutes a major health problem. Add to this the long duration of treatment advised and the need for regularity of treatment-if deformity is to be prevented and the risk of drug resistance minimised-and the world situation can be seen in its stark reality, which is reflected in successive reports from the World Health Organisation and its Expert Committee.

Those of us who have been concerned for upwards of 40 years with mass treatment schemes for leprosy and ambulatory treatment/control measures integrated into the general urban-rural health services cannot but agree that "sulphones provide a cheap and practical form of treatment and also prevent transmission of leprosy". A recent editorial in Leprosy Review says the same thing: "The maximal extension of sulphone treatment, and a skilled sustained campaign of leprosy education are priorities for us all." The problem is to discover large scale schemes, adequately documented and supervised, in which this thesis can be conclusively demonstrated. An oversimplification of his case, illustrated by atypical populations with "a small leprosy problem" and highly selective official estimates of incidence rates, may not convince the cautious epidemiologist.

It would be a salutary exercise to expose the deficiencies in Crawford's article: "the mode of transmission and the incubation period are unknown"; "there is no . . vector to spread the disease"; "only one in ten of the exposed population, at the most, acquire the disease"; leprosy declined in Norway "by a policy of segregation"; "once eradicated from any country, it has never returned"; "no attempt has been made to obtain information about incidence rates"; "accurate figures of leprosy are available ... in Rumania,
Cyprus and Libya".

From recent personal observations, we consider that the conclusions drawn by Crawford regarding the situation in some of the countries he refers to are not in accordance with the facts; on the contrary, the total prevalence rates (comprising the accumulated back-log of high incidence rates associated with a virtual breakdown of rural health services) are much higher than they were 15 or 20 years ago. This does not, of course, invalidate his main thesis that regular and adequate sulphone

\section{What kind of strategy for leprosy?}

By S. G. Browne, Director of the Leprosy Study Centre, and T. F. Davey, of Leprosy Revicw.

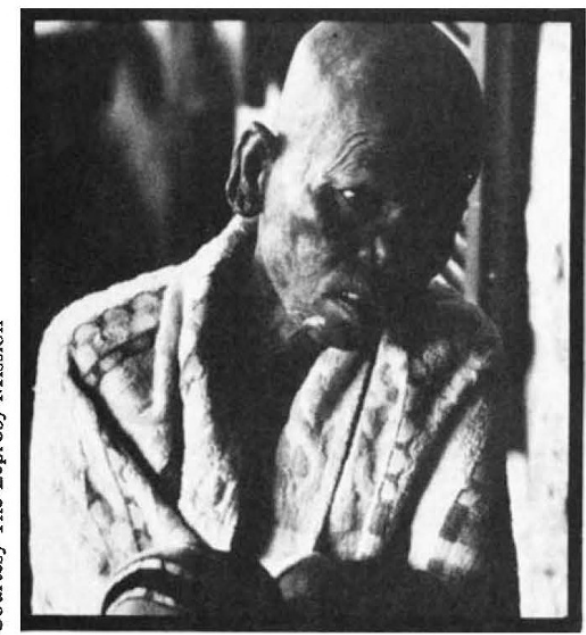

therapy could eventually result in a reduction of incidence rates, but it does emphasise the realities of today's world and the intractability of the problems of the control of transmissible diseases, among which leprosy figures along with malaria, tuberculosis, trypanosomiasis, onchocerciasis, schistosomiasis and the rest.

Crawford asscrts that "drug resistance is very rare". It was, but it is not now, and the recent figures coming from countries where the problem has been adequately investigated are very disturbing, to say the least.

"In many (countries) the leprosarium remains the only place where treatment can be obtained". This statement is hopelessly out-of-date, as is the incredible assertion that, of the $£ 2$ million raised annually by voluntary agencies in Europe for leprosy work, most is still spent on institutional care. More than 900,000 leprosy sufferers are being treated at the moment through programmes assisted financially by these maligned voluntary agencies, and the vast majority of these patients are being treated in ambulatory schemes; in addition, about $£ 250,000$ a year is being devoted to leprosy research. About a third of all leprosy patients now receiving any treatment at all are getting it through these same voluntary organisations. If such mis-statements are allowed to pass uncorrected, a grave disservice will be done to the cause of truth and to the leprosy sufferer.

As for prophylaxis, to judge by the tempo of much cooperative research based on the availability of relatively enormous quantitics of $M$. leprae obtained from experimentally infected armadillos, a specific vaccine should be produced in the not-too-distant future, together with a specific skin test. Chemoprophylaxis (with dapsone) has been shown to be effective, as Crawford points out, but here again the practical objections to large scale implementation are over-riding. Countries where such a measure might be justifiable on general grounds lack both the medical infrastructure and the finance, not to mention the cooperation of the population necessary over a prolonged period (possibly 3-5 years) during which the regular ingestion of a toxic drug by mouth (dapsone) or by intramuscular injection (acedapsone every 75 days) would be necessary.

Ministries of Health the world over are facing the problem of a chronic crippling disease, which, ideally and considered in isolation, might require a special service with vast resources-for diagnosis, laboratory cover, prolonged treatment (with a drug that is cheap, admittedly, but whose real cost is greatly enhanced by the expense of getting it to the patients who need it), preventive physiotherapy, provision of protective footwear, not to mention rehabilitation facilities. The social and medical dangers of over-emphasising one of the endemic diseases lie in creating or perpetuating the idea of 'uniqueness' and 'difference' and hence encouraging the stigma attached to the disease.

If treatment of leprosy could be rapid as well as cheap, unaccompanied by severe episodes of reaction as well as effective, capable of bacillary clearance as of mycobacteriostasis, then Crawford's fundamental thesis would be gladly accepted by governments. Mcanwhile, inadequately staffed and inadequately financed health services in many countries in the Third World, while awaiting a more effective strategy of control hased on primary prevention of leprosy, are striving with varying degrees of enthusiasm and success to put into practice the princinles of secondary prevention based on the availability of a cheap and reasonably effective drug. dapsone. 